\title{
Possibility of concentration of nonvolatile species near the surface of comet $67 \mathrm{P} /$ Churyumov-Gerasimenko
}

\author{
T. Suzuki ${ }^{1,2}$, Y. Shinnaka ${ }^{3}$, L. Majumdar ${ }^{4}$, T. Shibata ${ }^{2,5,6}$, Y. Shibaike ${ }^{7,8,9}$, H. Nomura ${ }^{2}$, and H. Minamoto ${ }^{7}$ \\ ${ }^{1}$ Astrobiology Center, Osawa 2-21-1, Mitaka, Tokyo 181-8588, Japan \\ e-mail: taiki.suzuki@nao.ac.jp \\ 2 National Astronomical Observatory of Japan, 2-21-1 Osawa, Mitaka, Tokyo 181-8588, Japan \\ ${ }^{3}$ Laboratory of Infrared High-resolution Spectroscopy (LiH), Koyama Astronomical Observatory, Kyoto Sangyo University, \\ Motoyama, Kamigamo, Kita-ku, Kyoto 603-8555, Japan \\ ${ }^{4}$ School of Earth and Planetary Sciences, National Institute of Science Education and Research, HBNI, Jatni 752050, Odisha, India \\ e-mail: liton@niser.ac.in \\ 5 The University of Tokyo, 7-3-1 Hongo, Bunkyo-ku, Tokyo 113-8654, Japan \\ ${ }^{6}$ Core Concept Technologies Inc., 11F DaiyaGate Ikebukuro, 1-16-15 Minamiikebukuro, Toshima-ku, Tokyo 171-0022, Japan \\ ${ }^{7}$ Department of Earth and Planetary Sciences, Tokyo Institute of Technology, 2-12-1 Ookayama, Meguro-ku, Tokyo 152-8551, \\ Japan \\ ${ }^{8}$ Physikalisches Institut \& NCCR PlanetS, Universitaet Bern, 3012 Bern, Switzerland \\ ${ }^{9}$ Earth-Life Science Institute, Tokyo Institute of Technology, Meguro, Tokyo 152-8550, Japan
}

Received 26 May 2020 / Accepted 2 December 2020

\begin{abstract}
Context. Cometary materials are thought to be the reservoir of the primitive materials of the Solar System. The recent detection of glycine and $\mathrm{CH}_{3} \mathrm{NH}_{2}$ by the ROSINA mass spectrometer in the coma of 67P/Churyumov-Gerasimenko suggests that amino acids and their precursors may have formed in an early evolutionary phase of the Solar System.

Aims. We aim to investigate the evolution of the interior of comets considering the evaporation process of water followed by the concentration of nonvolatile species.

Methods. We developed a Simplified Cometary Concentration Model (SCCM) to simulate the evaporation and concentration processes on the cometary surface. We use 67P/Churyumov-Gerasimenko as the benchmark of the SCCM. We investigated the depth of the layer where nonvolatile species concentrate after the numerous passages of perihelion after the formation of the Solar System.

Results. We find that the SCCM explains the observed production rates of water and $\mathrm{CH}_{3} \mathrm{NH}_{2}$ at 100 comet years. Results from the SCCM suggest that the nonvolatile species would concentrate in the top $100 \mathrm{~cm}$ of the comet surface within 10 comet years. Our results also suggest that the nonvolatile species would concentrate several meters beneath the surface before it hit the early Earth. This specific mass of nonvolatile species may provide unique chemical conditions for the volcanic hot spring pools.
\end{abstract}

Key words. comets: individual: 67P/Churyumov-Gerasimenko - methods: numerical - ISM: abundances - astrochemistry

\section{Introduction}

Since the pioneering suggestion by Oró (1961) of external delivery of organic materials by comets or meteorites, many researchers have investigated this possible supply of organic materials to the early Earth. Ehrenfreund et al. (2002) strengthened the case for comets as the sources of organic materials compared to other possible sources on Earth, such as UV photolysis or electron discharging in the ancient atmosphere. Assuming comets have indeed delivered organic materials to Earth, an attempt to understand the origins of life must necessarily begin with detailed studies of the formation and evolution of complex organic molecules in space. These organic molecules are the products of complex chemistry that most likely starts in molecular clouds and continues within the protoplanetary disk, which is the birthplace of comets and asteroids. Detections of various kinds of organic materials in the Murchison meteorites, such as sugars and amino acids (Cooper et al. 2001; Engel \& Nagy 1982), support this theory.

If this is the case, the chemical evolution of material is likely to start from the parent cloud of the Solar System. Recent observations improved our understanding of the chemical composition of the protoplanetary disk and star-forming regions. The high-sensitivity observation of ALMA achieved detections of the complex organic molecules $\mathrm{CH}_{3} \mathrm{CN}$ (Öberg et al. 2015; Loomis et al. 2018; Bergner et al. 2018), $\mathrm{CH}_{3} \mathrm{OH}$ (Walsh et al. 2016), and $\mathrm{HCOOH}$ (Favre et al. 2018). In addition, observations toward low-mass star-forming regions are much easier to carry out than those toward protoplanetary disks because of the high beam-averaged column densities of molecules. More complex molecules such as glycolaldehyde $\left(\mathrm{HCOCH}_{2} \mathrm{OH}\right)$ (Jørgensen et al. 2012), methyl isocyanate $\left(\mathrm{CH}_{3} \mathrm{NCO}\right)$ (Martin-Domenech et al. 2017), and $\mathrm{CH}_{3} \mathrm{Cl}$ (Fayolle et al. 2017) were reported toward low-mass star-forming regions. Glycine $\left(\mathrm{NH}_{2} \mathrm{CH}_{2} \mathrm{COOH}\right)$ in particular, the simplest and only nonchiral member of the 20 standard amino acids, has gathered the attention of astronomers; however, none of the observations were successful toward any star-forming regions (e.g., Ceccarelli et al. 2000). Astrochemical modeling of interstellar glycine led to the prediction that $\mathrm{CH}_{3} \mathrm{NH}_{2}$ and $\mathrm{CH}_{2} \mathrm{NH}$ are precursors of glycine (Garrod 2013; Suzuki et al. 2018a). Detections of $\mathrm{CH}_{3} \mathrm{NH}_{2}$ and $\mathrm{CH}_{2} \mathrm{NH}$ were reported toward various high-mass star-forming regions 
(Suzuki et al. 2018b; Ohishi et al. 2019). For glycine itself, Kuan et al. (2003) claimed the first detections of glycine toward highmass star-forming regions, but their detections were refuted by Snyder et al. (2005).

It is thought that the materials in star-forming regions and protoplanetary disks could be incorporated into comets. Walsh et al. (2014) performed a chemical modeling study for the protoplanetary disk and showed that grain-surface fractional abundances of simple and complex organic molecules (relative to water ice) for the outer disk are consistent with abundances derived for comets. Biver et al. (2015) also found good agreement between the chemical compositions of cometary coma and warm cores.

Amino acids are also a major target for in-situ and sample return missions on and from comets, respectively. Elsila et al. (2009) claimed the detection of glycine in the Stardust sample with its carbon isotopic ratio $\delta^{13} C=+29 \pm 6 \%$, suggesting an extraterrestrial origin. The latest paradigm shift of our understanding was brought about by the detailed analysis of comet 67P/Churyumov-Gerasimenko (hereafter 67P) by Rosetta mission, which enabled direct measurement of its chemical composition. This mission confirmed all the known chemical species at that time (Roy et al. 2015), and new detections of various complex species as well (e.g., $\mathrm{CH}_{3} \mathrm{NCO}, \mathrm{CH}_{3} \mathrm{COCH}_{3}, \mathrm{C}_{2} \mathrm{H}_{5} \mathrm{CHO}$, and $\mathrm{CH}_{3} \mathrm{CONH}_{2}$ ) through the direct analyses of the COmetary Sampling And Composition experiment COSAC (Goesmann et al. 2015), though the detection of $\mathrm{CH}_{3} \mathrm{NCO}$ was disputed by the proceeding study (Altwegg et al. 2017). Recent measurements suggest the existence of ammonium salt in this comet, which could be the substantial reservoir of nitrogen (Altwegg et al. 2020; Poch 2020). In addition, volatile glycine and its precursor, $\mathrm{CH}_{3} \mathrm{NH}_{2}$, were detected in the coma of comet 67P by the ROSINA (Rosetta Orbiter Spectrometer for Ion and Neutral Analysis) mass spectrometer (Altwegg et al. 2016). Hadraoui et al. (2019) argued that glycine would originate from sublimating water ice from dust particles that are ejected from the nucleus.

Though this latter scenario is fascinating, there is room for discussing the chemistry and physics of the cometary surface. As reported by Capaccioni et al. (2015), with the very low albedo on the surface of 67P, water rapidly sublimates from the surface, and refractory materials are left behind. Capaccioni et al. (2015) also suggested that refractory organic materials cover the comet surface. The Deep Impact mission (Mumma et al. 2005) reported a significant increase in the volatile species, $\mathrm{C}_{2} \mathrm{H}_{6}$, after impact with comet 9P/Tempel 1. The sudden increase of $\mathrm{C}_{2} \mathrm{H}_{6}$ implies that this volatile species was lost from the comet surface due to thermal processing, while it is still frozen below the surface of the comet. This observation also emphasizes the importance of thermal processing when discussing chemical composition close to the comet surface. During thermal processing by the Sun, volatile species would be easily lost, while nonvolatile species would concentrate at the surface, leading to inhomogeneous distributions of molecules.

Comets would have hit the early Earth, providing material. Pierazzo \& Chyba (1999) performed hydrocode simulations of the comet impact and studied the thermal decomposition rates of amino acids based on the time evolution of the temperature after impact. The simulations of these latter authors showed that amino acids on the opposite side to the collision point could survive the high-temperature conditions during the impact. If this were the case, it would provide the high-density organic mass without diluting to the volcanic hot spring pools, which are suggested as the candidate place for the origin of life (Damer

\section{(a) Evaporation Process}

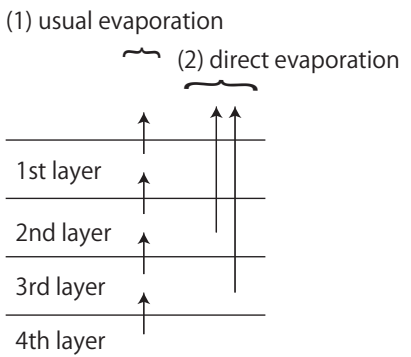

(b) Merging Process

\begin{tabular}{|c|c|c|c|}
\hline 1st layer & $P=0.8$ & 1st layer & $P=0.2$ \\
\hline 2nd layer & $P=0.4$ & 2nd layer & $P=0.2$ \\
\hline 3rd layer & $P=0.2$ & 3rd layer & $P=0.1$ \\
\hline
\end{tabular}

Fig. 1. Our SCCM model, which includes two evaporation mechanisms. In addition to the usual evaporation process to transport molecules toward the upper layer, we consider the direct evaporation process whose efficiency strongly depends on the porosity of upper layers. When the porosity exceeds 0.7 , the layer will merge with the layer beneath.

\& Deamer 2020). As the concentration of nonvolatile species provides the volcanic hot spring pools with a unique chemical composition, it is interesting to investigate the chemical distribution in the cometary interiors. In addition, a previous modeling study by Keller et al. (2015) overestimated the water production rate of 67P. The concentration of the nonvolatile species may reduce the evaporation of water and other species from the cometary interior, which may improve the agreement between the modeled and observed water sublimation rates.

In this paper, we present a new physical model for the evolution of the cometary interior, including the evaporation processes of water, $\mathrm{CH}_{3} \mathrm{NH}_{2}$, and nonvolatile species. In Sect. 2, we describe the details of the Simplified Cometary Concentration Model (SCCM). Then, in Sect. 3, we benchmark SCCM with the production rates of water and $\mathrm{CH}_{3} \mathrm{NH}_{2}$ and the thickness of the layer composed of nonvolatile species. We summarize our findings in Sect. 4.

\section{Simplified Cometary Concentration Model (SCCM)}

The SCCM takes into account the layered structure of a comet. Our layered structure is depicted in Fig. 1. The thickness of the layers is the parameter that determines the resolution of the vertical structure. Whilst using thinner layers improves the accuracy of the model, it requires higher computational time.

In the SCCM model, we calculate the temperature of the $n$th layer at time $t, T[n, t]$, with the following procedure: we take $t$ in "comet years", where one comet year is $6.45 \mathrm{yr}$ according to the JPL Small-Body Database Browser ${ }^{1}$, which corresponds to one orbital period of the comet 67P. First, we simulate the orbit of $67 \mathrm{P}$ with its orbital parameters to calculate the surface

1 https://ssd.jpl.nasa.gov/sbdb.cgi 
T. Suzuki et al.: Possibility of concentration of nonvolatile species near the surface of comet 67P/Churyumov-Gerasimenko
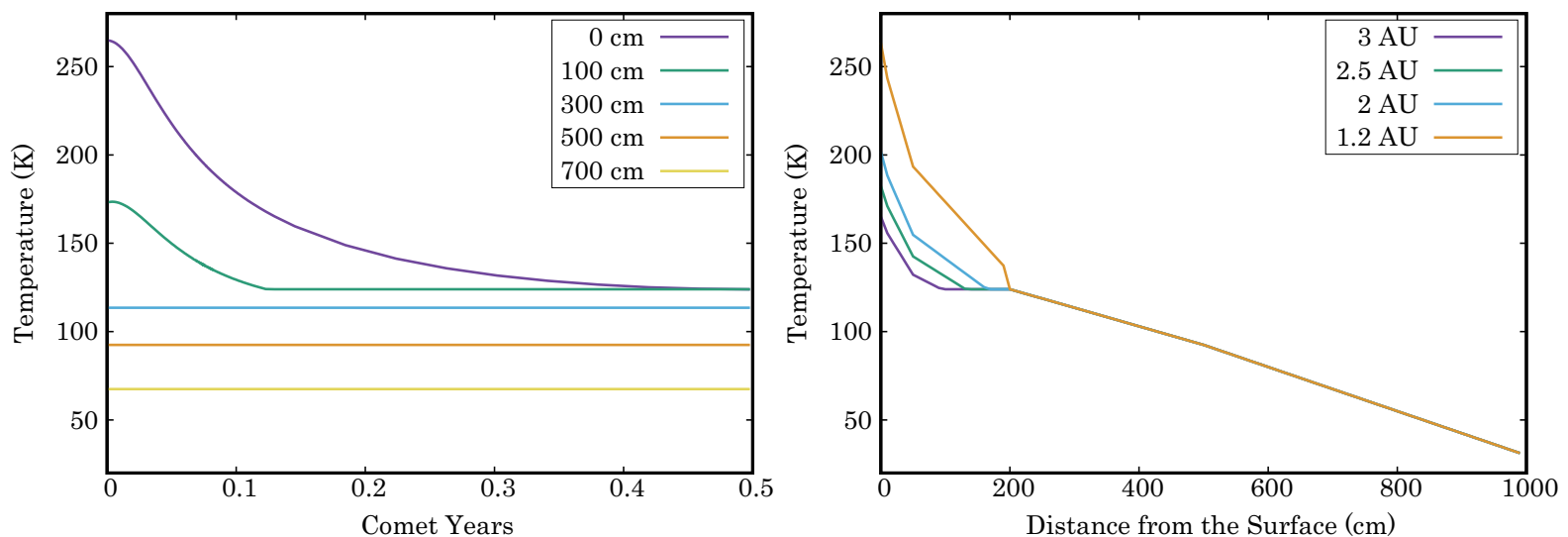

Fig. 2. Temperature distribution model for inside of the comet 67P/Churyumov-Gerasimenko. Left: vertical temperature distribution of the surface of 67P/Churyumov-Gerasimenko as a function of distance from the Sun. Right: temperature distribution at varying distance from the surface as a function of time in comet years. The time of $0 \mathrm{yr}$ corresponds to the position of perihelion.

temperature at time $t, T[n=1, t]$. We then assume radiative equilibrium between irradiation from the Sun and the blackbody radiation of the comet. The obtained temperature is in good agreement with that of Guilbert-Lepoutre et al. (2016) for the surface. For simplicity, we calculate the surface temperature only from zero to 0.5 comet years and assume the periodic time variation after that. We note that this symmetric temperature variation is not strictly correct, as the actual peak activity of 67P is observed two weeks after perihelion (Snodgrass et al. 2016). We use the temperatures of 260, 240, 190, and $120 \mathrm{~K}$ for the depths of $0,10,50$, and $200 \mathrm{~cm}$, respectively, at the perihelion $(t=0)$, following the calculation of Guilbert-Lepoutre et al. (2016). The peak temperatures at other depths, $T[n, t=0]$, were linearly interpolated. Following Guilbert-Lepoutre et al. (2016), we assume that the temperature is $120 \mathrm{~K}$ for all layers at 0.5 comet years, when the comet is the most distant from the Sun. Then, for depths of less than $200 \mathrm{~cm}$, we calculate the temperature of the $n$th layer at the time $t$ as

$T[n, t]=T[n=1, t] \times f[n, t]$,

where $f[n, t]=2 \times(1-q[n]) \times t+q[n]$ and $q[n]=T[n, t=$ $0] / T[n=1, t=0]$. We neglect the time variation of the temperature for the layers deeper than $200 \mathrm{~cm}$. We fix the temperature of 120 and $30 \mathrm{~K}$ at a depth of 200 and $1000 \mathrm{~cm}$, respectively, and use linear interpolation at other depths. There is no temperature gradient inside the single layer. We show our temperature distribution in Fig. 2.

We use temperature to calculate the evaporation rate from the comet surface with Eq. (2) of Hasegawa et al. (1992), which is frequently used in astrochemical models (Ruaud et al. 2016):

$t[n, j, t]_{\text {evaporation }}=v_{0}^{-1} \exp \left(E[j] / k_{\mathrm{B}} T[n, t]\right)$, where,

$v_{0}=\left(2 n_{\mathrm{s}} E[j] / \pi^{2} m\right)^{1 / 2}$.

The inverse of the evaporation timescale is the evaporation rate $k[n, j, t]$ (i.e., $k[n, j, t]=t[n, j, t]_{\text {evaporation }}^{-1}$ ). $E[j]$ is the binding energy, which is determined by the strength of the interaction of species $j$ with water ice, $n_{\mathrm{s}}$ is the surface density of the site, which is $1.5 \times 10^{15} \mathrm{~cm}^{-2}$ (Tielens \& Allamandola 1987), $m$ is the mass of the molecules, and $k_{\mathrm{B}}$ is the Boltzmann constant. The evaporation rate of species $j$ from the $n$th layer at time $t$, " $k[n, j, t]$ ", is proportional to $\exp \left(-E[j] / k_{\mathrm{B}} T[n, t]\right)$. Hence, the binding energy and the temperature are essential for determining $k[n, j, t]$. We assume that all molecules can evaporate from the surface without being trapped inside. While the molecules in the deeper layers have to move to upper layers one by one through this usual evaporation process (Eq. (5)), they can escape directly from the comet from any layer via a direct evaporation process as described in Eq. (6) below. The nonvolatile species should be mainly minerals and insoluble organic matter (Fray et al. 2017). The binding energies for $\mathrm{H}_{2} \mathrm{O}$ and $\mathrm{CH}_{3} \mathrm{NH}_{2}$ are 5700 and $6500 \mathrm{~K}$, respectively, based on the theoretical prediction by Wakelam et al. (2017). We assume that thermal evaporation is negligible for nonvolatile species. For glycine, the binding energy would be $13000 \mathrm{~K}$ (Tzvetkov et al. 2004; Suzuki et al. 2018a), and the thermal evaporation is not dominant in the orbit of 67P. Therefore, we treat glycine as a nonvolatile species.

In SCCM modeling, we define the porosity $P[n, t]$ as

$P[n, t]=\frac{V_{\text {Max }}-V_{\text {Real }}[n, t]}{V_{\text {Max }}}$,

where $V_{\text {Real }}[n, t]=\Sigma_{j} V_{\text {Real }}[n, j, t]$ represents the sum of the volumes occupied by materials of all species $j$ in the $n$th layer and at $t$, while $V_{\text {Max }}$ denotes the maximum volume in a certain layer. From this definition, it is apparent that the term $V_{\text {Real }}[n, t]=(1-P[n, t]) \times V_{\text {Max }}$ denotes the real volume occupied by any material in a layer. Here we introduce the ratio of certain species " $j$ " in the $n$th layer at $t$, that is, $r[n, j, t]$ as $r[n, j, t]=V_{\text {Real }}[n, j, t] / V_{\text {Real }}[n, t]$. The sum of the ratios of all molecules, $\sum_{j} r[n, j, t]$, should always be unity.

With the term of $V_{\text {Real }}[n, j, t]=V_{\text {Real }}[n, t] \times r[n, j, t]=(1-$ $P[n, t]) \times V_{\mathrm{Max}} \times r[n, j, t]$, the evaporated volume amount of species $j$ from the first layer $(n=1)$ during the time step $\Delta t$, $V_{\text {evap }}[1, j, t]$, is given by

$\frac{\Delta V_{\text {evap }}[1, j, t]}{\Delta t}=k[1, j, t] \times V_{\text {Real }}[1, j, t]$.

As the porosity $P[1, t]$ represents the percentage of cavity, $(1-P[1, t]) \times V_{\text {Max }}$ represents the actual amount of material in the first layer, that is, the surface of the comet" or similar. The volume amount of material transferred from $n+1$ th layer to $n$th layer during the time step $\Delta t$ is calculated as

$$
\begin{aligned}
\frac{\Delta V_{\text {evap }}[n+1, j, t]}{\Delta t}= & P[n, t] \times k[n+1, j, t] \\
& \times V_{\text {Real }}[n+1, j, t] \quad(n \geqq 1) .
\end{aligned}
$$


Although the default time step $\Delta t$ is $1000 \mathrm{~s}$, the time step is half that value when (1) the evaporation rate of at least one species $j, \Delta t \times k[n, j, t]$, is less than 1 , and (2) the molecular ratio of species $j, r[n, j, t]$, is more than $1 \%$. The first criteria will enable us to perform accurate calculation of volatile species, especially $\mathrm{H}_{2} \mathrm{O}$. When $\Delta t \times k[n, j, t]$ exceeds 1 and $r[n, j, t]$ is less than $1 \%$, the term of $\Delta t \times k[n, j, t]=1$, corresponding to the situation that all material is transferred to the upper layer during $\Delta t$. Similar to the equation for the evaporation process from the first layer, $k[n+1, j, t] \times V_{\text {Real }}[n+1, j, t]$ denotes the total volume amount of thermal evaporation for species $j$. However, this equation represents the blocking of material transfer to the upper layer by multiplying the porosity of the upper layer, $P[n, t]$ (Fig. 1).

We assume that a part of the material can evaporate directly from any layer if there is enough porosity in the upper layer (Fig. 1). The volume amount of direct evaporation for species $j$ from the $n$th layer is presented as

$\Delta V_{\text {direct }}[n+1, j, t]=\left(\prod_{k=1}^{k=n} P[k, t]\right) \times \Delta V_{\text {evap }}[n+1, j, t]$.

By multiplying the term of $\prod_{k=1}^{k=n} P[k, t]$, this equation provides a means by which to evaporate materials directly from the inner layer when the porosity of the upper layers is high enough.

The evaporation processes (5) to (7) determine the volume amount of lost material from the layer. The differentiation of porosity and molecular ratios can be calculated as the result of the above processes. The differentiation of $V_{\text {Real }}$ during the time-step $\Delta t$ can be calculated as

$$
\begin{aligned}
\Delta V_{\text {Real }}[n, j, t]= & -\Delta t \times\left(\Delta V_{\text {evap }}[n, j, t] / \Delta t\right. \\
& \left.-\Delta M_{\text {evap }}[n+1, j, t] / \Delta t+\Delta V_{\text {direct }}[n, j, t] / \Delta t\right) .
\end{aligned}
$$

Then, $V_{\text {Real }}$ after the time-step $\Delta t$ is presented as

$V_{\text {Real }}[n, j, t+\Delta t]=V_{\text {Real }}[n, j, t]+\Delta V_{\text {Real }}[n, j, t]$.

Then, recalling the definition of porosity, new porosity $P[n, t+$ $\Delta t]$ is calculated as

$P[n, t+\Delta t]=\frac{V_{\mathrm{Max}}-\Sigma_{j} V_{\mathrm{Real}}[n, j, t+\Delta t]}{V_{\mathrm{Max}}}$.

As $V_{\text {Real }}[n, j, t]=(1-P[n, t]) \times r[n, j, t] \times V_{\text {Max }}$, this equation is independent of $V_{\mathrm{Max}}$. The time evolution of molecular ratios is calculated as

$$
\begin{aligned}
& r[n, j, t+\Delta t]=V_{\text {Real }}[n, j, t+\Delta t] / V_{\text {Real }}[n, t+\Delta t] \\
& \quad=V_{\text {Real }}[n, j, t]+\Delta V_{\text {Real }}[n, j, t] /(1-P[n, t+\Delta t]) \times V_{\text {Max }} .
\end{aligned}
$$

Finally, we consider merging layers when the porosity gets larger than 0.7 after the above calculations. This value of 0.7 is determined considering the observed porosity of 67P (Pätzold et al. 2016). In addition, the sum of the total volume of layers, $V_{\text {Real(before) }}[n, t]+V_{\text {Real(before) }}[n+1, t]$, must be smaller than $V_{\text {Max }}$ before the merging process so that the volume of the new layer does not exceed the limitation after merging. Then, using the definition of porosity, the merging process is considered when $P[n, t]+P[n+1, t]>1$ is achieved. With these restrictions, new molecular ratios and the porosity for the $n$th layer can be calculated from the definition of porosity. We fix $V_{\text {Max }}$ during merging but the real volume of material is regarded as the sum of the volumes of the original two layers.

$$
\begin{aligned}
P_{\text {new }}[n, t] & =\frac{V_{\text {Max }}-\left(V_{\text {Real(before) }}[n, t]+V_{\text {Real(before) }}[n+1, t]\right)}{V_{\text {Max }}} \\
& =\left(P_{\text {before }}[n, t]+P_{\text {before }}[n+1, t]\right)-1 .
\end{aligned}
$$

Then, new molecular ratios can be calculated as

$$
\begin{aligned}
& r_{\text {new }}[n, j, t] \\
& =\frac{\left(1-P_{\text {before }}[n, t]\right) r[n, j, t]+\left(1-P_{\text {before }}[n+1, t]\right) r[n+1, j, t]}{1-P_{\text {new }}[n, t]} .
\end{aligned}
$$

After the merging process, we reassign the layer number to compensate the merged layer. In this process, the old $n+2$ th layer is regarded as new $n+1$ th layer by applying $r_{\text {new }}[n+1, j, t]=$ $r_{\text {before }}[n+2, j, t]$ and $P_{\text {new }}[n+1, t]=P_{\text {before }}[n+2, t]$. The conservation of the total volume of material is kept with the above equations.

The timescale of SCCM is also essential because nonvolatile species would efficiently concentrate after the long timescale of the heating by the Sun. Ip et al. (2016) calculated the history of the orbital evolution of $67 \mathrm{P}$ due to the gravitational interactions between giant planets. These latter authors suggest that the perihelion of comet 67P changed suddenly in 1959 from 2.7 to 1.3 AU. Before 1959, the perihelion was between 2 and 3 au for $300 \mathrm{yr}$, but it would have the perihelion of $\sim 1.2$ au between about 300 and $900 \mathrm{yr}$ ago. Therefore, this comet would have experienced strong heat processing by the solar flux for several hundred years. To simulate such a long thermal processing timescale, we calculated the time evolution of the porosity and the molecular ratios during 100 comet years. In Sect. 4, we also discuss the case where the comet 67P did not experience heating before 1959 .

Finally, our model is highly simplified in many ways. Although the photo-dissociation process may destroy the molecules, we do not include it in the SCCM modeling. According to the experimental result, molecules would be protected from solar UV photons because these latter can only penetrate to thousands of nanometers (Barnett et al. 2012). However, solar and cosmic particles may affect our results by destroying molecules. In addition, we neglect sideways and inward diffusion. While sideways diffusion will be canceled by diffusion in the opposite direction, inward diffusion may delay the concentration process. As we see in the following section, the concentration process is almost complete within 10 comet years. Therefore, the delay would not be particularly important if we assume the longer timescale. Our model does not consider the effect of the rotation of $67 \mathrm{P}$ either.

\section{SCCM results}

\subsection{Parameter dependency}

We performed SCCM simulations under the six different sets of chemical ratios, sizes of layers, and initial porosity. These parameters are summarized in Table 1 . We include $\mathrm{H}_{2} \mathrm{O}$ and $\mathrm{CH}_{3} \mathrm{NH}_{2}$, and nonvolatile species. As water is the dominant component of cometary gas, it is used to simulate the concentration mechanism of nonvolatile species. In addition, we include $\mathrm{CH}_{3} \mathrm{NH}_{2}$ as representative of less-volatile species, whose binding energy is higher than water, to test its behavior during the calculation. We also note that $\mathrm{CH}_{3} \mathrm{NH}_{2}$ is known as a precursor of glycine. 
Table 1. Parameters for SCCM modeling.

\begin{tabular}{lccc}
\hline \hline Model & $\begin{array}{c}\text { Initial ratios of nonvolatile } \\
\text { species }\end{array}$ & $\begin{array}{c}\text { Layer size } \\
(\mathrm{cm})\end{array}$ & Initial porosity \\
\hline Set 1 & 0.5 & 10 & 0 \\
Set 2 & 0.5 & 10 & 0.2 \\
Set 3 & 0.5 & 5 & 0 \\
Set 4 & 0.5 & 20 & 0 \\
Set 5 & 0.1 & 10 & 0 \\
Set 6 & 0.9 & 10 & 0 \\
\hline
\end{tabular}

Notes. We show the initial volume occupied by nonvolatile species, layer thickness, and porosity.

Though the list of included species is limited, they will provide us enough information to discuss the concentration mechanism.

We set the initial porosity of zero for Set 1 . Though the porosity of zero is much lower than suggested by observations of $67 \mathrm{P}$, we use this porosity to assume an ideal comet, which is free from heat processing, for the initial condition. We set the volume ratio of nonvolatile species to $50 \%$. Other materials are mainly water, but $\mathrm{CH}_{3} \mathrm{NH}_{2}$ is also included in our model so that we may study the behavior of molecules with binding energies higher than water. Considering the simulated abundance ratio of $\mathrm{CH}_{3} \mathrm{NH}_{2} / \mathrm{H}_{2} \mathrm{O}$ on interstellar grains, we set the ratio of $\mathrm{CH}_{3} \mathrm{NH}_{2}$ to be $0.1 \%$ (Suzuki et al. 2018a). All layers have the same initial molecular ratios regardless of the depth.

We use Sets 2 to 6 to investigate the parameter dependency of the SCCM. We change the initial porosity from zero to 0.2 in Set 2 while the other parameters are kept the same as in Set 1. The averaged porosity and the time evolution of the chemical composition of Set 2 (Figs. 3 through 6) show good agreement with Set 1. Therefore, the initial porosity does not affect our results.

The layer thickness is another parameter of the SCCM model. We set the layer thickness to be 5 and $20 \mathrm{~cm}$ in Sets 3 and 4, respectively, while the other parameters are kept the same as in Set 1. As a result, the time evolution of the porosity and the chemical ratios (Figs. 3 through 6) show a small difference, especially in the later phase ( $~ 90$ comet years) of the simulation. With these results, we fix the porosity to be zero and the layer thickness to be $10 \mathrm{~cm}$ in the following discussion.

\subsection{Simulation results}

In this section, we discuss the evolution of the comet interior under the assumption of different dust-to-ice ratios. As it is difficult to estimate the initial dust-to-ice ratio of the comet before the thermal processing of the Sun, the initial dust-to-ice ratio of SCCM is determined from the observation of the comet 67P. Fulle et al. (2017) suggested a high dust-to-ice ratio of 7.5, while other studies reported a lower dust-to-ice ratio of between 0.6 and 7 (Choukroun et al. 2020, and references therein). Therefore, we test three models assuming the different ratios of nonvolatile species. In Sets 1, 5, and 6, respectively, the ratio of nonvolatile species is assumed to be 10,50 , and $90 \%$. We set a layer thickness of $10 \mathrm{~cm}$ and an initial porosity of zero for all cases.

In Sets 1,5 , and 6, the averaged porosity in $0-60,60$ 120 , and $120-180 \mathrm{~cm}$ from the surface get to $\sim 0.5, \sim 0.6$, and $\sim 0.1$ within 15 comet years (see Fig. 3). The averaged ratios at the different depth beneath the comet surface are shown in Figs. 4-6. At the depths of 0-60 and 60-120 cm, water evaporates very quickly. The porosity at $0-60$ and $60-120 \mathrm{~cm}$ is almost 0.5 , the ratios of $\mathrm{H}_{2} \mathrm{O}$ and $\mathrm{CH}_{3} \mathrm{NH}_{2}$ are zero, and the ratio of nonvolatile species is unity throughout the simulation due to the rapid evaporation of $\mathrm{H}_{2} \mathrm{O}$. The evaporation of $\mathrm{H}_{2} \mathrm{O}$ leads to the concentration of nonvolatile species (Fig. 6) for all sets. Though the temperature is slightly high for the upper layer, the porosity is low for the deeper layer. As a result, $\mathrm{H}_{2} \mathrm{O}$ evaporates from the deeper layer and is frozen on the upper layer when the comet is away from the Sun, which increases the $\mathrm{H}_{2} \mathrm{O}$ ratio while it decreases the porosity and the nonvolatile species ratio. On the other hand, the $\mathrm{H}_{2} \mathrm{O}$ ratio decreases when the comet approaches the Sun. This behavior causes oscillation in the porosity and the ratios. In addition, the dynamic time evolution of the porosity and ratios is seen in Set 5, where the initial ratio of $\mathrm{H}_{2} \mathrm{O}$ is 0.9 . The complete evaporation of $\mathrm{H}_{2} \mathrm{O}$ will lead to porosity of more than 0.7 , and the merging process of layers leads to a sudden change in the porosity and the ratios. On the other hand, the merging process is not essential for Set 6 , where the ratio of $\mathrm{H}_{2} \mathrm{O}$ is only 0.1 . With this small ratio, the evaporation of $\mathrm{H}_{2} \mathrm{O}$ has less of an effect on the merging process. The porosity increases slowly once the ratio of nonvolatile species increases.

The layers beneath more than $180 \mathrm{~cm}$ take more time to lose $\mathrm{H}_{2} \mathrm{O}$ due to the lower temperature. The average porosity at 240-300 cm in depth (the twenty-fourth to thirtieth layer) still changes even after 100 comet years. The evaporation of $\mathrm{H}_{2} \mathrm{O}$ is still triggering the merging process in this region. The difference of $\mathrm{CH}_{3} \mathrm{NH}_{2}$ ratio in Sets 1 and 5 at $240-300 \mathrm{~cm}$ below the surface is due to the higher abundance of nonvolatile species in Set 1 .

\section{Discussion}

\subsection{Water production rate}

As water is the dominant compound of comets, the production rate of water would be the best benchmark with which to test the SCCM. In our model, the production rate of the $j$ th species at time $t$, Production $[j, t]$, is given by summing the total volume of evaporation from the surface $(n=1)$ and the indirect evaporation from the deeper layer.

$$
\begin{aligned}
\text { Production }[j, t]= & \rho \times S \times\left(\frac{\Delta V_{\text {evap }}[n=1, j, t]}{\Delta t}\right. \\
& \left.+\Sigma_{n \geqq 1} \frac{\Delta V_{\text {direct }}[n \geqq 1, j, t]}{\Delta t}\right) \times d \mathrm{~kg} \mathrm{~s}^{-1},
\end{aligned}
$$

where $\rho$ is the mean density of comet $67 \mathrm{P}$, corresponding to $470 \mathrm{~kg} \mathrm{~m}^{-3}$ (Sierks et al. 2015), $d$ is the layer thickness, and $S$ is the total surface area of the comet surface. Assuming that $67 \mathrm{P}$ is a sphere with a radius of $2 \mathrm{~km}, S$ is $5 \times 10^{7} \mathrm{~m}^{2}$. We calculate the water production rate of Sets 1,5 , and 6 at 100 comet years to compare SCCM results with the result of the Rosetta mission. We show the water production rate of Sets 1, 5, and 6 in Fig. 7 by the function of the distance to the Sun. The water production rate in August 2014 was $1.2 \mathrm{~kg} \mathrm{~s}^{-1}$ at 3.6 au (Gulkis et al. 2015), while the maximum value is $1.73 \times 10^{2} \mathrm{~kg} \mathrm{~s}^{-1}$ after perihelion (Bertaux et al. 2015).

The peak production rates of water in Sets 5, 1, and 6 are 447,73 , and $7.7 \mathrm{~kg} \mathrm{~s}^{-1}$, while they are $11,1.7$, and $0.2 \mathrm{~kg} \mathrm{~s}^{-1}$ at 3.6 AU. The result of Set 6 underestimates the production 

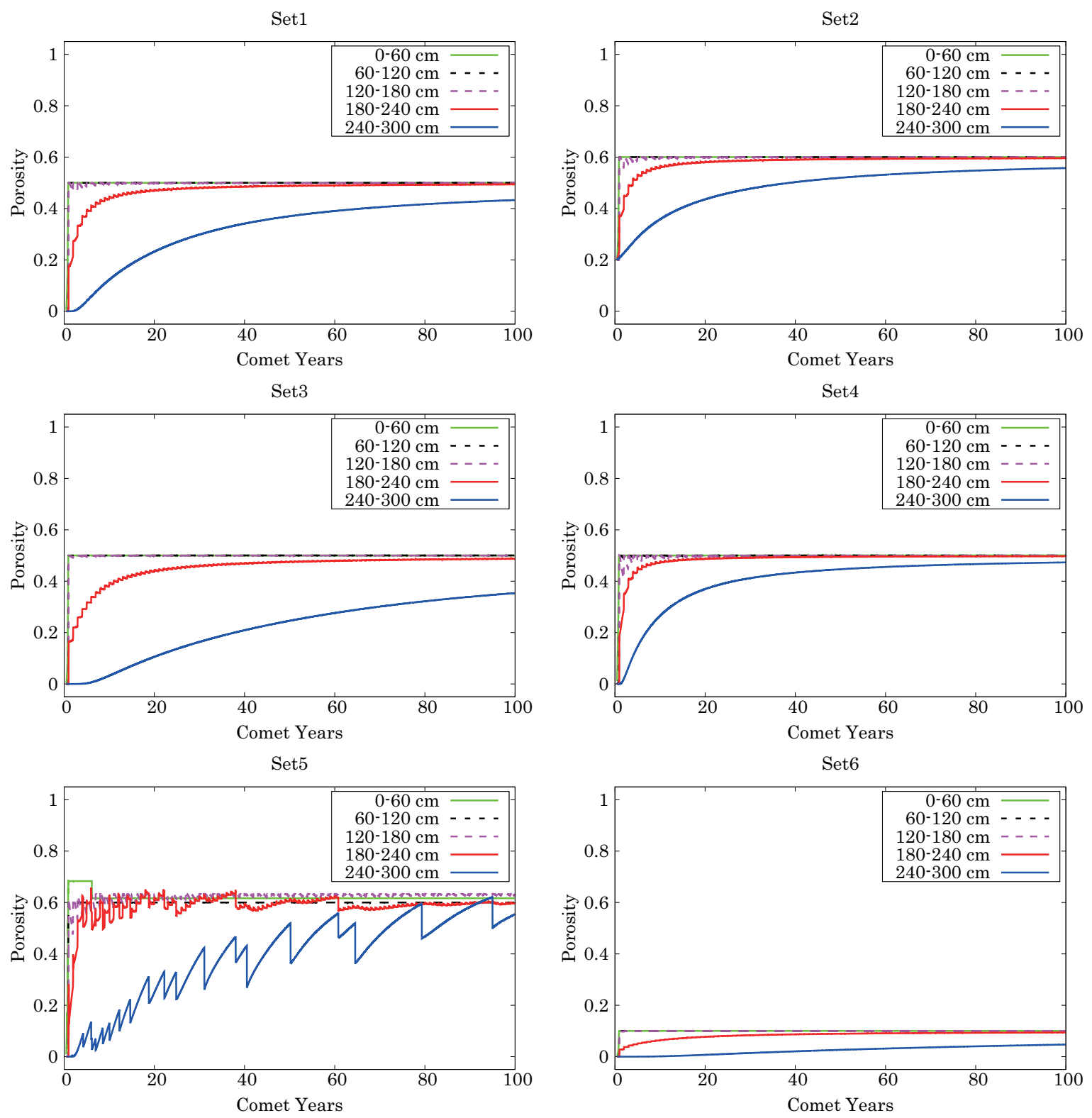

Fig. 3. Evolution of averaged porosity for the different depths. The lines represent the depths of $0-60,60-120,120-180,180-240$, and $240-300 \mathrm{~cm}$ with different colors.

rate. The production rate of Set 1 shows good agreement with observations.

It would be worth comparing our model with the previous theoretical modeling study of Keller et al. (2015). These latter authors simulated the detailed morphology of 67P, but the thickness of the layer of nonvolatile species was assumed to be of micrometer scale. According to their model B, the water production rate at $3.6 \mathrm{AU}$ was $61 \mathrm{~kg} \mathrm{~s}^{-1}$, which is higher than the observation by Gulkis et al. (2015) by an order of magnitude. Our agreement on the water production rate emphasizes the validity of the SCCM. We evaluated the erosion depth with our model by summing up the production rate along with an orbit followed by division by the density.

Erosion $[j]=\Sigma_{t} \frac{\text { Production }[j, t] \Delta t}{\rho} \mathrm{m}$.

We find erosion depths of 0.3, 0.03, and $0.006 \mathrm{~m}$ for Sets 1, 5, and 6, respectively, at 100 comet years. These depths are smaller than those found by Keller et al. (2015), where the erosion depths were between 0.67 and 2.9 .

\subsection{Chemical composition}

We compare the water production rates obtained by Eq. (14) with the observation of the Rosetta mission. The production rate ratio, $\mathrm{CH}_{3} \mathrm{NH}_{2} / \mathrm{H}_{2} \mathrm{O}$, is $0.007,0.004$, and 0.002 for Sets 1, 5, and 6 , respectively, when the distance to the Sun is more than 2 AU. These ratios agree well with the observed ratio of 0.006 (Goesmann et al. 2015).

\subsection{Timescale of the heating}

If comet 67P did not experience any heating before 1959, the heating timescale of $67 \mathrm{P}$ would be only eight comet years. We show the water production rates at this age with Sets 1,5 , and 6 in Fig. 7. At this early phase of the thermal processing, the water 

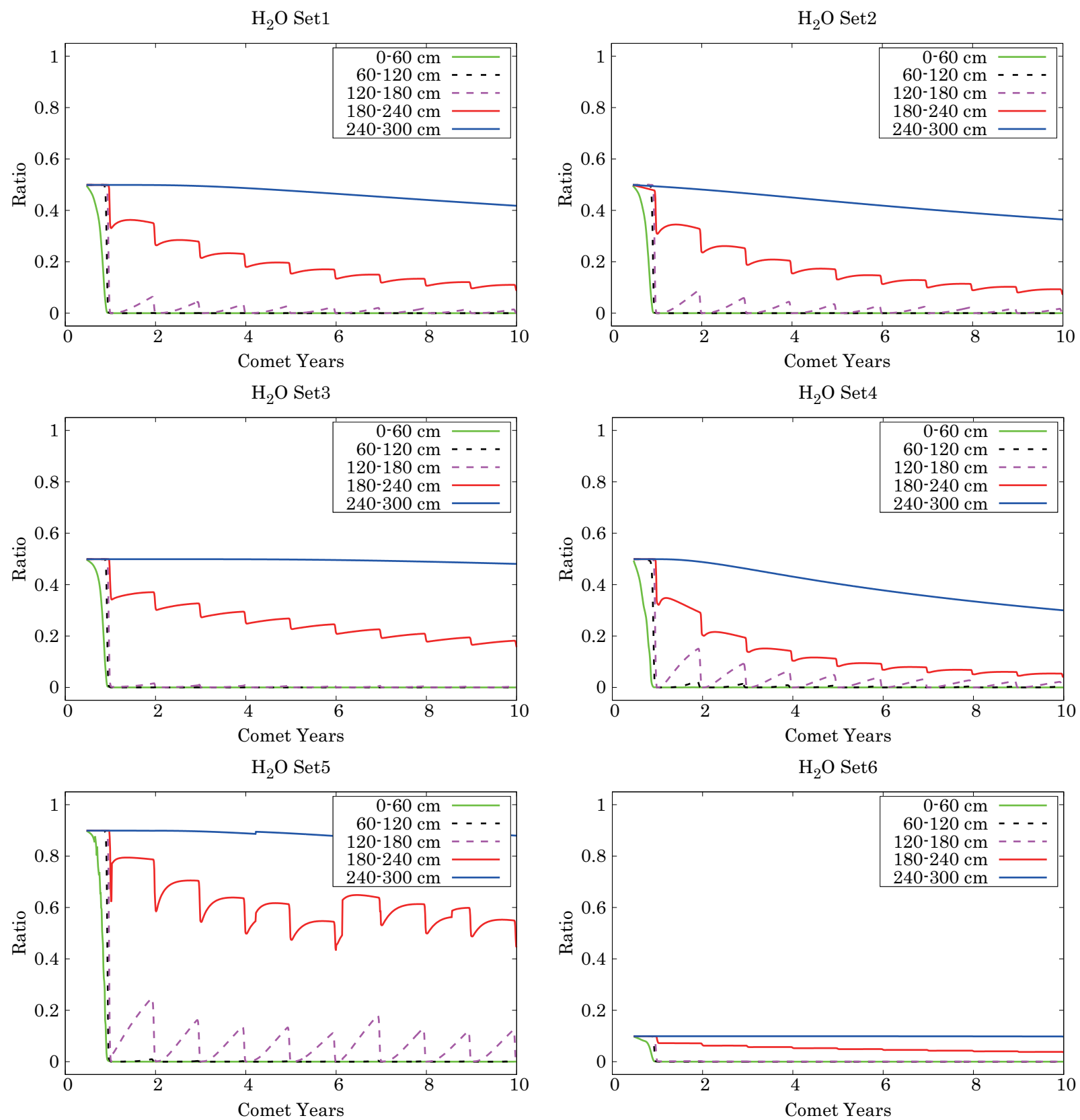

Fig. 4. With six sets, the time evolution of $\mathrm{H}_{2} \mathrm{O}$ ratio compared to the total volume occupied by materials is shown. The sum of the ratios of water, $\mathrm{CH}_{3} \mathrm{NH}_{2}$, and nonvolatile species is unity. The different colors represent the depths of 0-60, 60-120, 120-180, 180-240, and 240-300 cm. The horizontal axis represents the time in comet years, with one unit being the one orbital motion around the Sun.

production rates for Sets 1 and 5 are as high as $\sim 10^{3} \mathrm{~kg} \mathrm{~s}^{-1}$, as there is abundant water near the comet surface. The water production rate for Set 6 is $\sim 10 \mathrm{~kg} \mathrm{~s}^{-1}$ for the perihelion, while it is $\sim 0.6 \mathrm{~kg} \mathrm{~s}^{-1}$ at $3 \mathrm{au}$. Therefore, the SCCM model can explain the water production rate if we assume that the ratio of the nonvolatile species is high for the interior of $67 \mathrm{P}$. We note that the $\mathrm{CH}_{3} \mathrm{NH}_{2} / \mathrm{H}_{2} \mathrm{O}$ ratio does not dramatically change with the history of the thermal processing. Without any information on the $\mathrm{H}_{2} \mathrm{O}$ ratio inside 67P, it would be difficult to constrain its history of thermal processing.

\subsection{Possible effect on the chemistry in the coma}

Comet activity may release concentrated materials to the coma. Rosetta observed 34 outbursts within three months surrounding its perihelion passage, suggesting this event is common at least for 67P (Vincent et al. 2016). As these outbursts are characterized by observable changes in luminosity, small comet activities that we are not able to observe should be more ubiquitous. Several mechanisms for comet activity would be crystallization of amorphous ice, enrichment of volatile species inside the comet, and receding fractured cliffs (e.g., Agarwal et al. 2017; Vincent et al. 2016).

Altwegg et al. (2016) suggested a correlation of glycine abundance with dust density, suggesting the possibility that glycine does not directly originate from the nucleus of the comet but is evaporated from dust particles ejected by comet activity. The dust particles are efficiently heated by solar flux (Lien 1990), resulting in a higher temperature and thus release of the absorbed glycine. Hadraoui et al. (2019) also showed that glycine could 

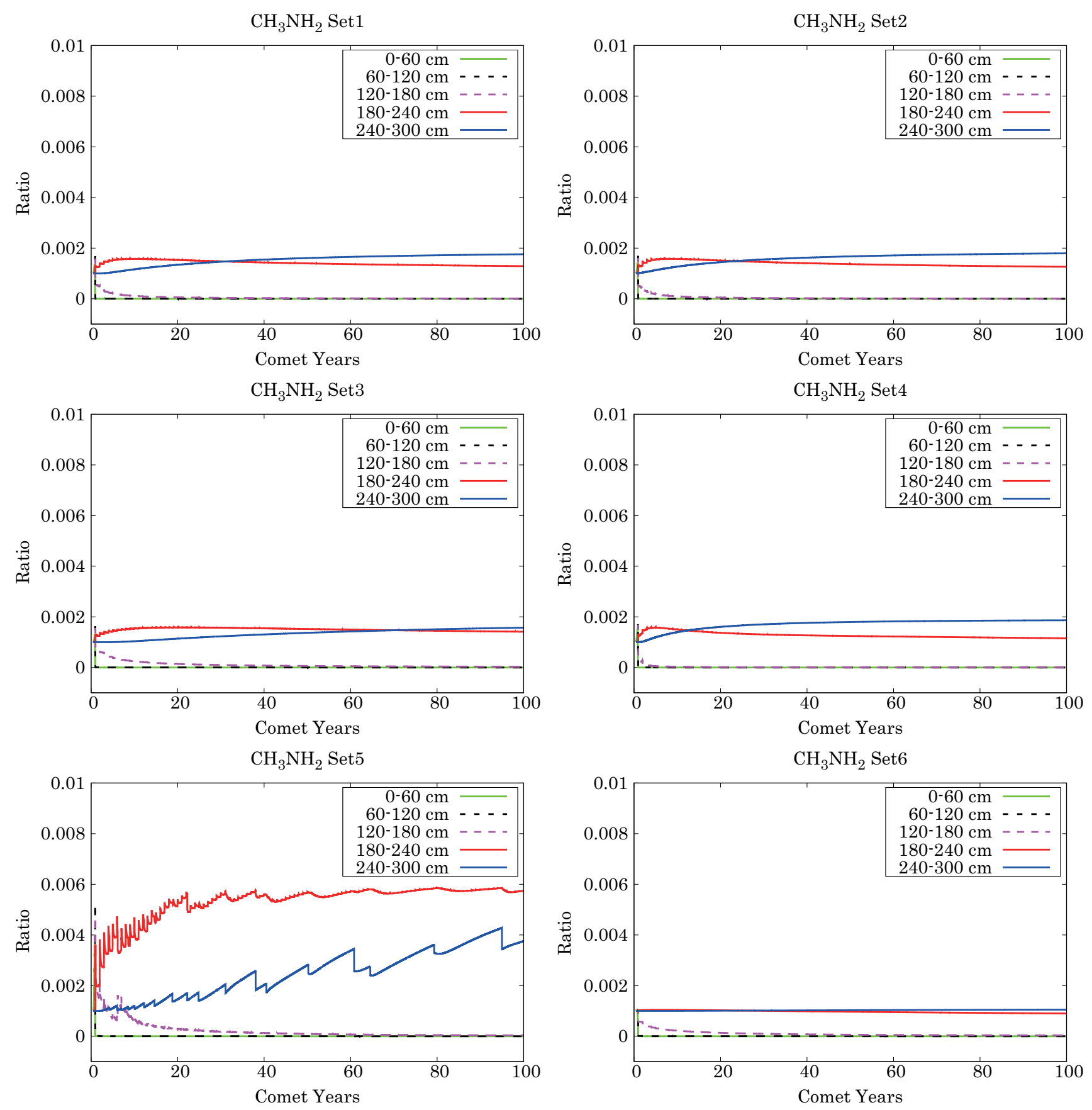

Fig. 5. Same as Fig. 4 but with $\mathrm{CH}_{3} \mathrm{NH}_{2}$.

originate from icy dust particles with water ejected from the nucleus. Though these studies do not consider the concentration of the nonvolatile species, the release of nonvolatile species from the comet surface can affect the observed molecular abundances in the coma.

\subsection{Effect on the early Earth chemistry}

It is believed that the comets would have hit the early Earth, providing the materials for the origin of life (Ehrenfreund et al. 2002). Concentration of materials, perhaps in micrometeorites of cometary origin, would provide the unique chemical conditions of the hot volcanic spring pools that have been proposed to lead to the chemical evolution toward life. However, the survival

rate of organic materials during the cometary bombardment, especially for the large comets, is still controversial. Pierazzo \& Chyba (1999) investigated the thermal decomposition rates of amino acids based on the time evolution of the temperature after the impact with their hydrocode simulations. These authors showed that the time evolution of the temperature after the impact strongly depends on the position of molecules on the comet upon impact. The opposite side of the comet to the impact position is less heated and provides a higher possibility of survival of amino acids.

Figure 3 shows that the timescale of the concentration process for $67 \mathrm{P}$ is very short. The concentration of nonvolatile species at depths of between 0 and $100 \mathrm{~cm}$ would be achieved within ten comet years, which corresponds to $65.7 \mathrm{yr}$. As we can 

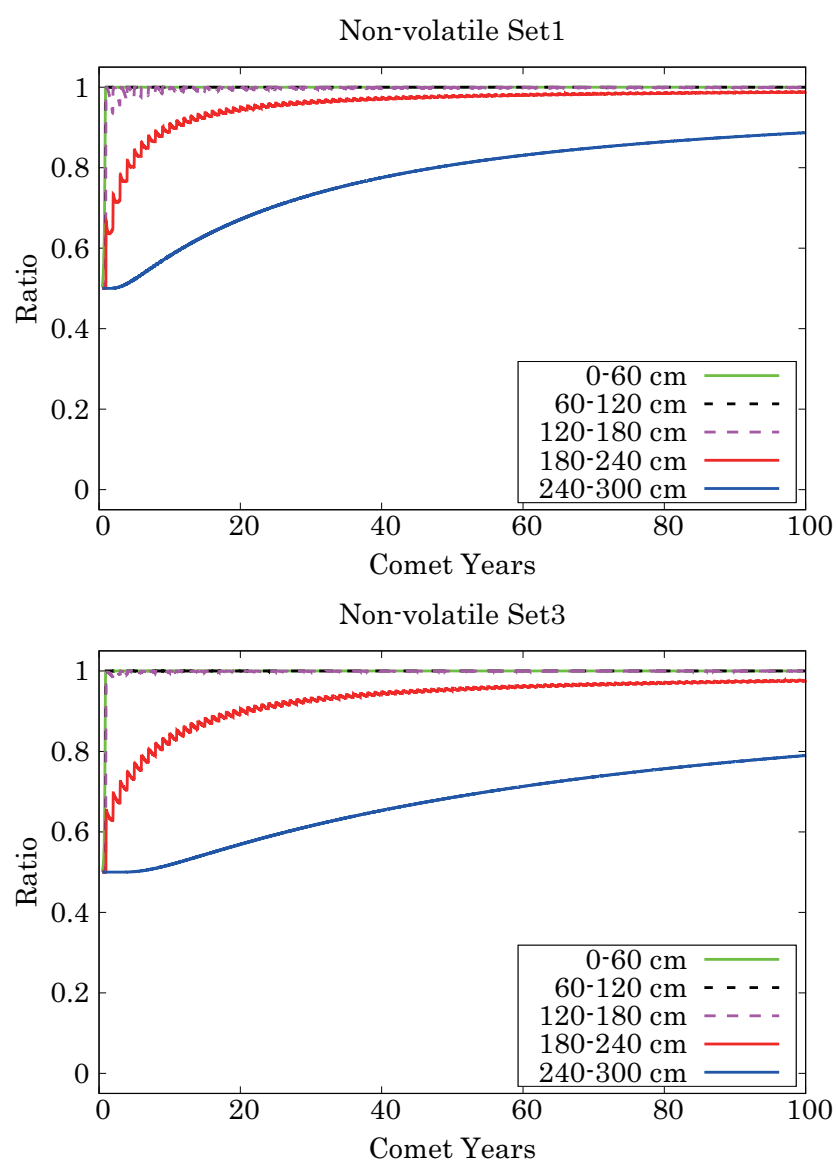

Non-volatile Set5

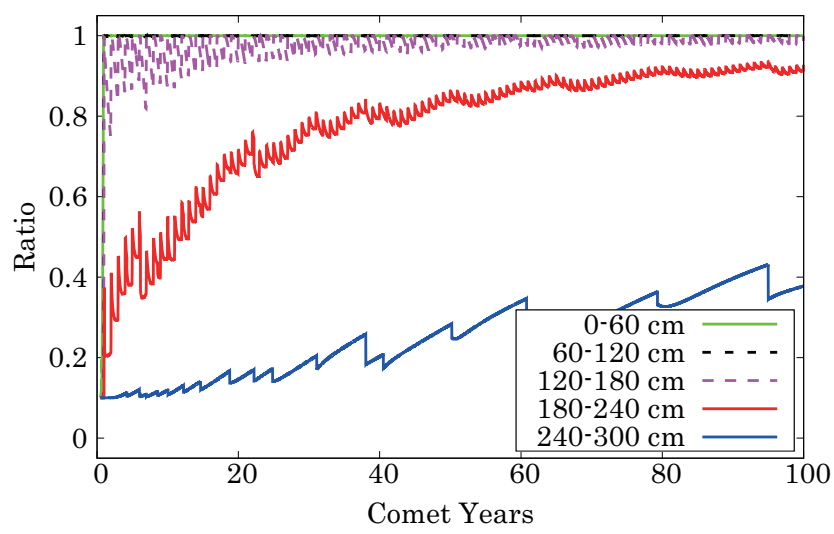

Fig. 6. Same as Fig. 4 but with nonvolatile species.

see from the concentration process for the deeper layers in Fig. 3, the typical depth of this concentration would be several meters beneath the surface. As the timescale of the concentration process is very short, most comets are expected to have hit the early Earth after concentration of nonvolatile species on the comet surface. The nonvolatile species would include amino acids and other complex and large molecules, which may be essential for chemical evolution toward the origin of life on Earth. Also, the concentration of these materials at the outer layer of comets is intriguing as the opposite side of the comet to the impact position would show lower temperature, where molecules are likely to survive. The outer layers on the opposite side of the comet are therefore of interest for further studies.
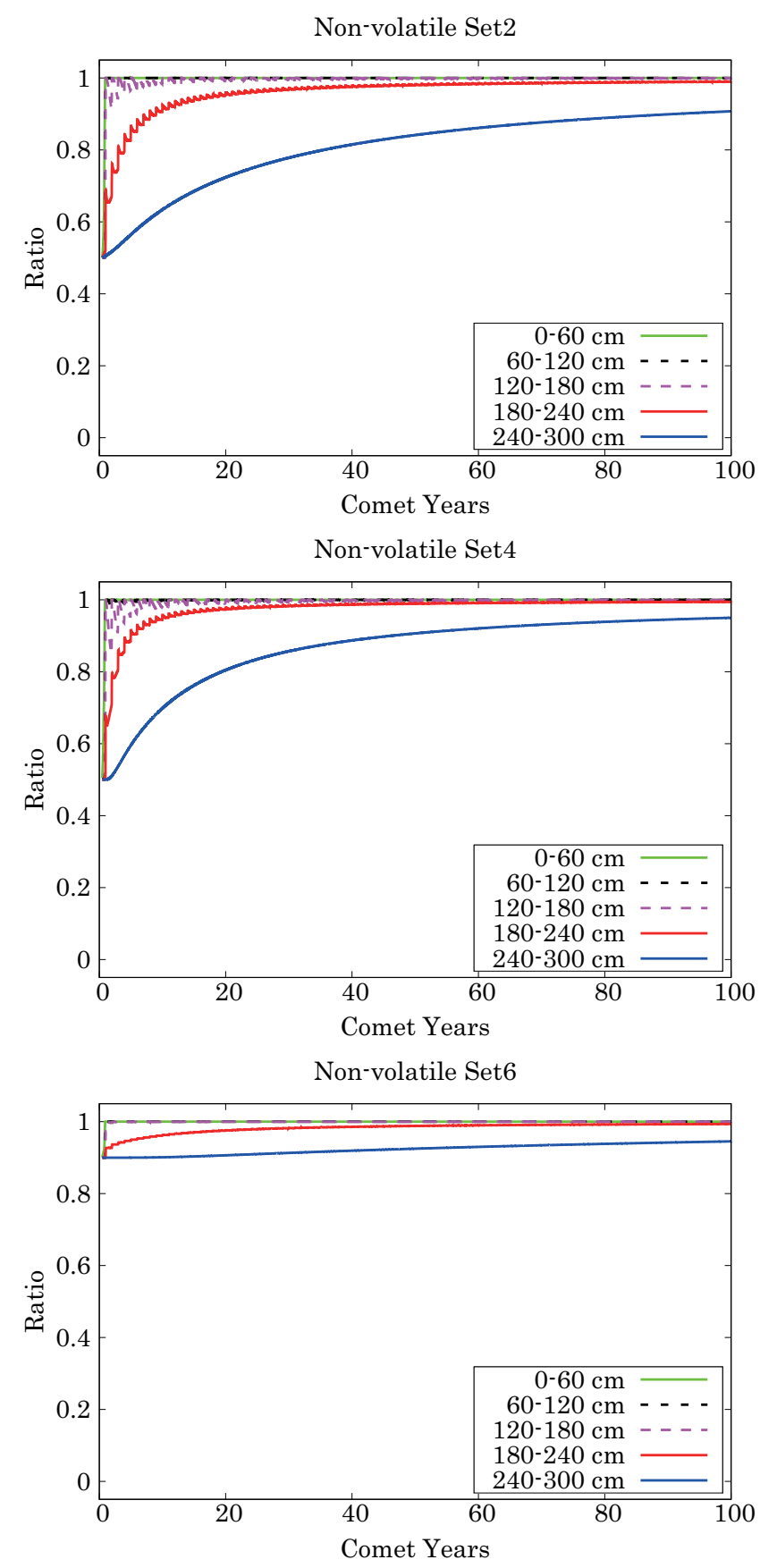

\section{Summary}

We investigated the evolution of the chemical composition of the outer cometary layers after heat processing by the Sun. We developed the SCCM model to study the importance of the concentration process of nonvolatile species, simulating the evaporation and the merging processes along with the orbit of comet 67P. We found that the SCCM model successfully reproduced the water production rate and the $\mathrm{CH}_{3} \mathrm{NH}_{2} / \mathrm{H}_{2} \mathrm{O}$ ratio for 67P. The SCCM model suggests that nonvolatile species may concentrate quickly near the comet surface, while the initial ratio remains the same deep inside comets. Comets may have a layer of nonvolatile species several meters below the surface when 

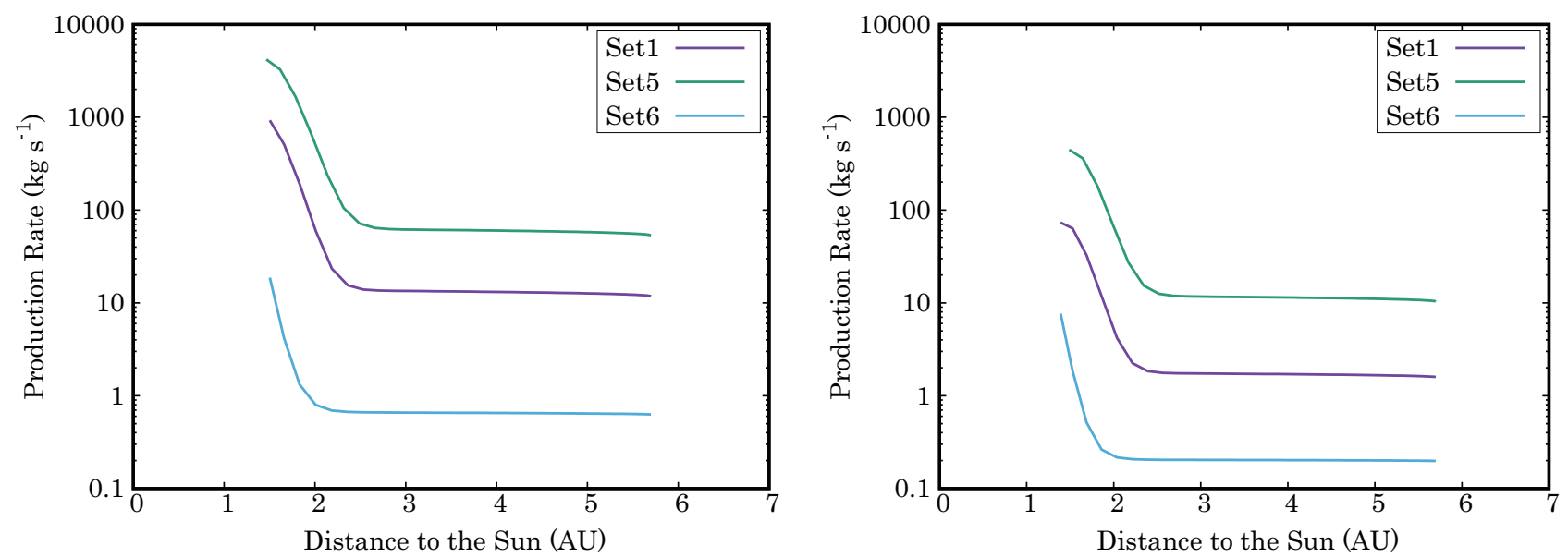

Fig. 7. Left: water production for Sets 1,5 , and 6, at seven comet years are depicted. This represents the specific case where 67P did not experience heating before 1959. Right: water production for Sets 1,5, and 6 at 100 comet years. This case simulates the thermal processing of the surface of $67 \mathrm{P}$ for the long timescale beyond 1959.

they hit the early Earth. The outer layer of the comet is of interest for further studies as the hot volcanic spring pools found there may provide unique chemical conditions that could be the origin of life on Earth.

Acknowledgements. We would like to thank the anonymous referee for constructive comments that helped to improve the manuscript. We are grateful to Dr. Takefumi Ootsubo and Dr. Hideyo Kawakita for the discussion. Parts of this work has been carried out within the frame of the National Centre for Competence in Research PlanetS supported by the Swiss National Science Foundation (SNSF). Y.S. acknowledges the financial support of the SNSF. This work was supported by JSPS KAKENHI Grant Number 20K14541, 19K03936, JP16J09590, 15J10864, and 15H02065.

\section{References}

Agarwal, J., Corte, V. D., Feldman, P. D., et al. 2017, MNRAS, 469, S606 Altwegg, K., Balsiger, H., Bar-Nun, A., et al. 2016, Science, 2, e1600285 Altwegg, K., Balsiger, H., Berthelier, J. J., et al. 2017, MNRAS, 469, S130 Altwegg, K., Balsiger, H., Hänni, N., et al. 2020, Nat. Astron. 4, 533

Barnett, I. L., Lignell, A., \& Gudipati, M. S. 2012, ApJ, 747, 13

Bergner, J. B., Guzmán, V, G., Öberg, K. I., Loomis, R., A., \& Pegues, J. 2018, A\&A, 857, 16

Bertaux, J.-L. 2015, A\&A 583, A38

Biver, N., Bockelée-Morvan, D., Moreno, R., et al. 2015, Sci. Adv., 1, e1500863

Bockelée-Morvan, D., Lis, D., Wink, J., et al. 2000, A\&A, 353, 1101

Capaccioni, F., Coradini, A., Filacchione, et al. 2015, Science, 347, 6220

Ceccarelli, C., Loinard, L., Castets, A., Faure, A., \& Lefloch, B. 2000, A\&A, 362,1122

Choukroun, M., Altwegg, K., Kührt, E., et al. 2020, Space Sci. Rev. 216, 44

Cooper, G., Kimmich, N., Belisle, et al. 2001, Nature, 414, 879

Damer, B., \& Deamer, D. 2016, Astrobiology, 20, 429

Elsila, J. E., Glavin, D. P., \& Dworkin, J. P. 2009, Meteorit. Planet. Sci., 44, 1323

Ehrenfreund, P., Irvine, W., Becker, L., et al. 2002, Rep. Prog. Phys. 65, 1427

Engel, M. H., \& Nagy, B. 1982, Nature, 296, 29

Favre, C., Fedele, D., Semenov, D., et al. 2018, ApJ, 862, L2

Fayolle, E. C., Öberg, K. I., Jøgensen, J. K. 2017, Nat. Astron., 1, 703

Fray, N., Bardyn, A., Cottin, H., et al. 2017, MNRAS, 469, S506
Fulle, M., Corte, V. D., Rotundi, A., et al. 2017, MNRAS, 469, S45

Garrod, R. T. 2013, ApJ, 765, 60

Goesmann, F., Rosenbauer, H., \& Bredehöft, J. H., et al. 2015, Science, 349, 6247

Guilbert-Lepoutre, A., Rosenberg, E. D., Prialnik, D., \& Besse, S. 2016, MNRAS, 462, S146

Gulkis, S., Allen, M., Allmen, P., et al. 2015, Science, 347, aaa0709

Hadraoui, K., Cottin, H., Ivanovski, S. L., et al. 2019, A\&A, 630, A32

Hasegawa, T. I., Herbst, E., \& Leung, C. M. 1992, ApJS, 82, 167

Ip, W.-H., Lai, I.-L., Lee, J.-C., et al. 2016, A\&A, 591, A132

Jørgensen, J. K., Favre, C., Bisschop, S. E., et al 2012, ApJ, 757, L4

Kellar, H. U., Mottola, S., Davidsson, B., et al. 2015, A\&A, 583, A34

Kuan, Y. J., Charnley, S. B., Huang, H. C., Tseng, W. L., \& Kisiel, Z. 2003 ApJ, 593,848

Lien, D. J. 1990, ApJ, 355, 680

Loomis, R. A., Cleeves, L. I., Öberg, K. I., et al. 2018, A\&A, 859, 11

Martín-Doménech, R., Rivilla, V. M., \& Jimenez-Serra, I. 2017, MNRAS 469 2230

Mumma, M. J., DiSanti, M. A., Magee-Sauer, K., et al. 2005, Science, 310, 270

Öberg, K. I., Guzman, V. V., Furuya, K., et al. 2015, Nature, 520, 198

Ohishi, M., Suzuki, T., Hirota, T., Saito, M., \& Kaifu, N. 2019, PASJ, 71, 86

Oró, P. J. 1961, Nature, 4774, 389

Pätzold, M., Andert, T., Hahn, M., et al. 2016, Nature, 530, 63

Pierazzo, E., \& Chyba, C. F. 1999, Meteor. Planet. Sci. 34, 909

Poch, O., Istiqomah, I., \& Quirico, P., et al. 2020, Science, 367, eaaw7462

Roy, L. L., Altwegg, K., Balsiger, H., et al. 2015, A\&A, 583, A1

Ruaud, M., Wakelam, V., \& Hersant, F. 2016, MNRAS, 459, 3756

Sierks, H., Barbieri, C., Lamy, P. L., et al. 2015, Science, 347, 1044

Snodgrass, C., Opitom, C., Val-Borro, M., et al. 2016, MNRAS, 462, S138

Snyder, L. E., Lovas, F. J., Hollis, J. M., et al. 2005 ApJ, 619, 914

Suzuki, T., Majumdar, L., Ohishi, M., et al. 2018a, ApJ, 863, 51

Suzuki, T., Ohishi, M., Majumdar, L. 2018b, ApJS, 237, 3

Tielens, A. G. G. M., \& Allamandola, L. J. 1987, Astrophys. Space Sci. Lib., 134,397

Tzvetkov, G., Ramsey, M. G., \& Netzer, F, P. 2004, Chem. Phys. Lett., 397, 392

Vincent, J.-B., A'Hearn, M. F., Lin, Z.-Y., et al. 2016, MNRAS, 462, S184

Wakelam, V., Loison, J. C., Mereau, R., \& Ruaud, M. 2017, Mol. Astrophys., 6, 22

Walsh, C., Millar, T. J., Nomura, H., et al. 2014, A\&A, 563, A33

Walsh, C., Loomis, R. A., Öberg, K. I., et al. 2016, ApJ, 823, L10 